\title{
In vivo photoacoustic flowmetry in the optical diffusive regime based on saline injection
}

Yong Zhou, Joemini Poudel, Guo Li, Lihong V. Wang

Yong Zhou, Joemini Poudel, Guo Li, Lihong V. Wang, "In vivo photoacoustic flowmetry in the optical diffusive regime based on saline injection," Proc. SPIE 9708, Photons Plus Ultrasound: Imaging and Sensing 2016, 97084 U (15 March 2016); doi: 10.1117/12.2217468

SPIE. Event: SPIE BiOS, 2016, San Francisco, California, United States 


\title{
In vivo photoacoustic flowmetry in the optical diffusive regime based on saline injection \\ Yong Zhou, Joemini Poudel, Guo Li, and Lihong V. Wang* \\ Optical Imaging Laboratory, Department of Biomedical Engineering, Washington University in \\ St. Louis, 1 Brookings Drive, Campus Box 1097, St. Louis, Missouri 63130 \\ *Correspondence: lhwang@wustl.edu
}

\begin{abstract}
We propose a saline-injection-based method to quantify blood flow velocity in vivo with acoustic-resolution photoacoustic tomography. By monitoring the saline-blood-interface propagating in the blood vessel, we can resolve the flow velocity. In phantom experiments, a root-mean-squared error of prediction of $0.29 \mathrm{~mm} / \mathrm{s}$ was achieved. By injecting saline into a mouse tail vein covered with $1 \mathrm{~mm}$ chicken tissue, we showed that the flow velocity in the tail vein could be measured at depth, which is especially pertinent to monitoring blood flow velocity in patients undergoing intravenous infusion.
\end{abstract}

Keywords: saline injection, blood flow velocity, acoustic-resolution photoacoustic tomography, intravenous infusion

\section{INTRODUCTION}

Photoacoustic tomography (PAT) is a modality that provides imaging in either two dimensions (2D) or three dimensions (3D). ${ }^{1-5}$ Combining the advantages of optical excitation and acoustic detection, PAT can image rich optical absorption contrast in biological tissues at depth. To date, PAT has been widely used for both structural and functional biological imaging in many different fields, including hematology, ${ }^{6}$ oncology, ${ }^{7}$ dermatology, ${ }^{8}$ ophthalmology, ${ }^{9}$ and gastroenterology. ${ }^{10}$ Depending on the limiting factor for spatial resolution, PAT can be divided into optical-resolution PAT (OR-PAT) and acoustic-resolution PAT (AR-PAT). In OR-PAT, the optical focus is much tighter than the acoustic focus, and a high spatial resolution can be achieved. AR-PAT provides a lower spatial resolution, defined by the acoustic focus. Nevertheless, because in biological tissue ultrasound suffers much less scattering than light, AR-PAT can achieve deep imaging with a depth-to-resolution ratio of more than $100 .{ }^{1}$ So far, with high resolution, OR-PAT has imaged a variety of important biological parameters in vivo, such as the oxygen saturation of hemoglobin $\left(\mathrm{sO}_{2}\right),{ }^{11}$ blood flow velocity, ${ }^{12,13}$ pulse wave velocity, ${ }^{14}$ and the metabolic rate of oxygen $\left(\mathrm{MRO}_{2}\right){ }^{9}$ However, although AR-PAT has imaged $\mathrm{sO}_{2}$ at depths, it still cannot provide in vivo blood flow information. In addition, in order to calculate $\mathrm{MRO}_{2}$ with PAT, flow needs to be measured, which makes it even more important to quantify flow.

There are two fundamental reasons why it is difficult for AR-PAT to measure blood flow velocity. First, unlike ultrasound, PAT has almost no speckles. ${ }^{15,16}$ If the target has a smooth boundary with respect to the wavelengths of the PA waves, the boundary signals of the target will stand out, while the speckles inside the target are largely suppressed. Because blood vessels in biological tissues usually have smooth boundaries, it is challenging for PAT to extract blood flow information based merely on speckle fluctuations. Second, AR-PAT has a lower spatial resolution than OR-PAT and thus a larger detection voxel size. In typical OR-PAT, the spatial resolution is comparable to the size of red blood cells (RBCs). Thus, when RBCs flow into and out of the detection voxel, the PA signal changes are observable. By monitoring how fast the signal changes ${ }^{17}$ the flow velocity can be calculated. However, in AR-PAT, the large detection voxel contains many more RBCs than in OR-PAT. Because the number of RBCs inside the detection voxel can be assumed to follow a Poisson distribution, ${ }^{18}$ a larger mean number of RBCs leads to a smaller relative RBC number change and thus a smaller PA signal change. For example, if there are $10,000 \mathrm{RBCs}$ in the detection voxel, the PA signal change due to the RBC number change would be only around $1 \%$, so the system would need a signal-to-noise (SNR) of more than 100 to measure the flow velocity.

Although challenging, different methods have been proposed to achieve blood flow measurement with AR-PAT, including PA Doppler (PAD) flowmetry ${ }^{19,20}$ and ultrasonically encoded PA flowgraphy (UE-PAF) ${ }^{21}$. Based on the

Photons Plus Ultrasound: Imaging and Sensing 2016, edited by Alexander A. Oraevsky, Lihong V. Wang Proc. of SPIE Vol. 9708, 97084U · (C) 2016 SPIE · CCC code: 1605-7422/16/\$18 · doi: 10.1117/12.2217468 
PAD effect, different PAD shifts have been observed from particles moving with different flow speeds, and the Doppler effect allows the flow speeds to be calculated. However, to observe the PAD shift, the moving particles have to be very sparse. Thus, this method cannot measure the flow velocity of whole blood. But by using ultrasound to encode the PA signals, UE-PAF can achieve whole blood flow imaging in deep tissue. In UE-PAF, modulated ultrasound is focused into the blood vessel to create a source of heat. Because PA signals are proportional to temperature, the PA signals from the heated area will increase. By monitoring the increased PA signals along the blood vessel, the flow speed in the blood vessel can be measured. However, this method's complexity has limited it to only phantoms, and so far, no in vivo data have been reported.

In this paper, we present a new method to measure blood flow velocity deep in biological tissues. To increase the PA signal changes due to flowing RBCs, saline is injected into the blood stream. As a result, at the saline-blood interfaces, the PA signals have sharp changes - blood's PA signal is strong, but saline's signal is negligibly low. Thus, by monitoring the time course of the PA signals from the interface, the flow velocity in the blood stream can be quantified. In addition, because saline is widely used for intravenous infusion, no extra saline injection is needed in such patients.
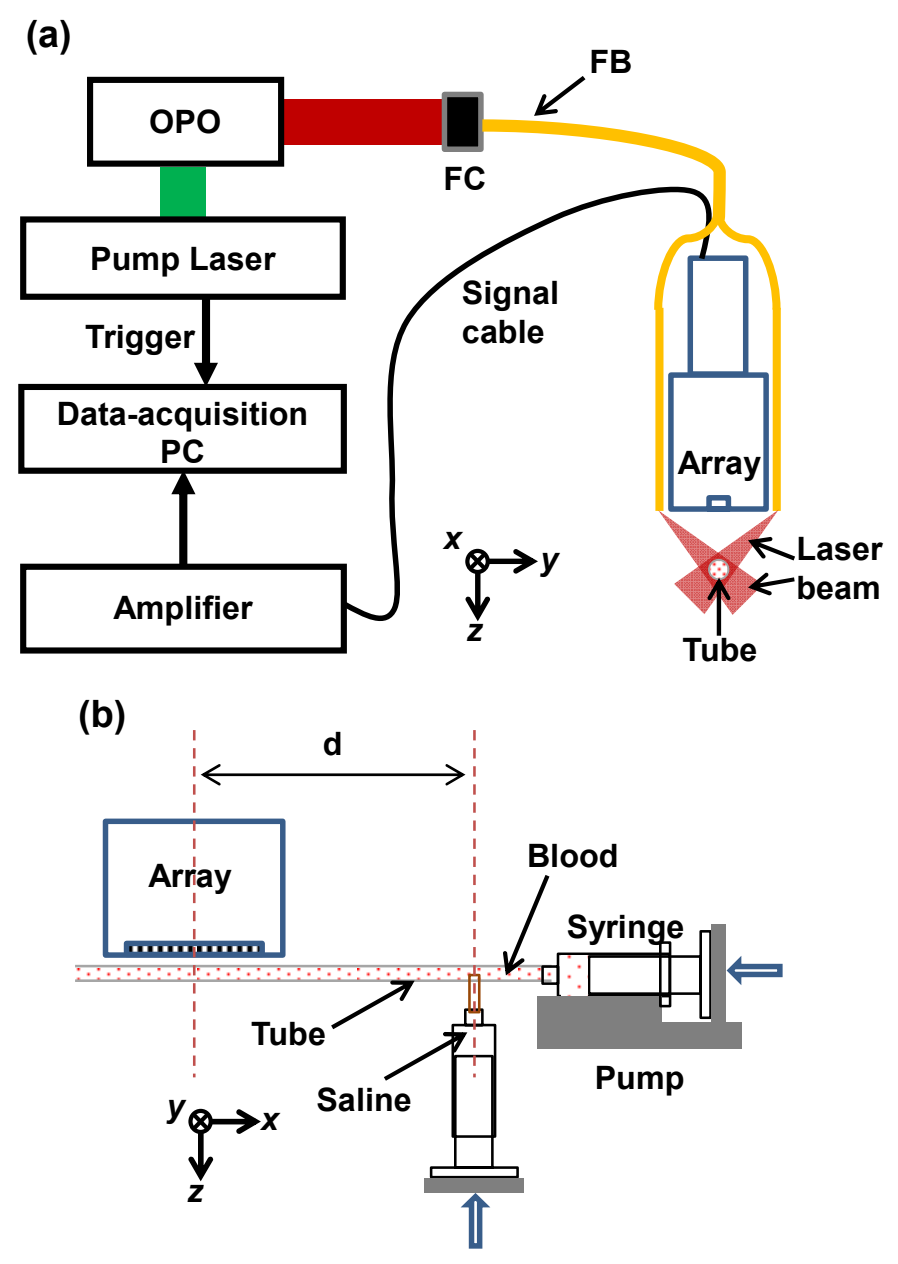

Fig. 1. Schematic of the photoacoustic tomography system (a) and saline injection (b). (a) BS, beam splitter; CL, cylindrical lens; FB, fiber bundle; FC, fiber coupler; M, mirror; OPO, optical parametric oscillator. (b) $\mathrm{d}$ was $15 \mathrm{~cm}$ in the phantom experiment. 


\section{MATERIALS AND METHODS}

We employed a typical AR-PAT system ${ }^{22-24}$ to demonstrate this idea. As shown in Fig. 1(a), the light source was a tunable optical parametric oscillator laser with a repetition rate of $20 \mathrm{~Hz}$. For deep tissue imaging, $680 \mathrm{~nm}$ light was chosen. The light was first coupled into an optical fiber bundle and then delivered onto the tissue by two rectangular strips at the output end of the fiber bundle. The incident pulse fluence on the tissue surface was controlled to be around $10 \mathrm{~mJ} / \mathrm{cm}^{2}$, which was less than the safety limit set by the American National Standards Institute $\left(20 \mathrm{~mJ} / \mathrm{cm}^{2}\right)$ at this wavelength. A linear-array transducer containing 256 elements (LZ250, Visualsonics Inc.) was used to detect the PA signals. Thus, each laser pulse generated a two-dimensional (2D) PA image. With a central frequency around $21 \mathrm{MHz}$ and a 78\% one-way bandwidth, this transducer array had an axial resolution of $86 \mu \mathrm{m}$. With a size of 23 $\mathrm{mm} \times 3 \mathrm{~mm}$ and a cylindrical focus at $15 \mathrm{~mm}$, this transducer array provided a lateral resolution of $119 \mu \mathrm{m}$ and an elevational resolution of $1237 \mu \mathrm{m}^{7}$

Figure 1(b) shows a schematic of saline injection in phantom experiments. A silicone tube filled with blood was used to mimic the blood vessel. The blood was pumped into the tube through a syringe, and the flow speed was controlled by a syringe pump. A second syringe was employed to perpendicularly inject saline solution into the tube. The distance between the injection point and the center of the field of view was set to $15 \mathrm{~cm}$ so the injection disturbance to the flow was minimized.
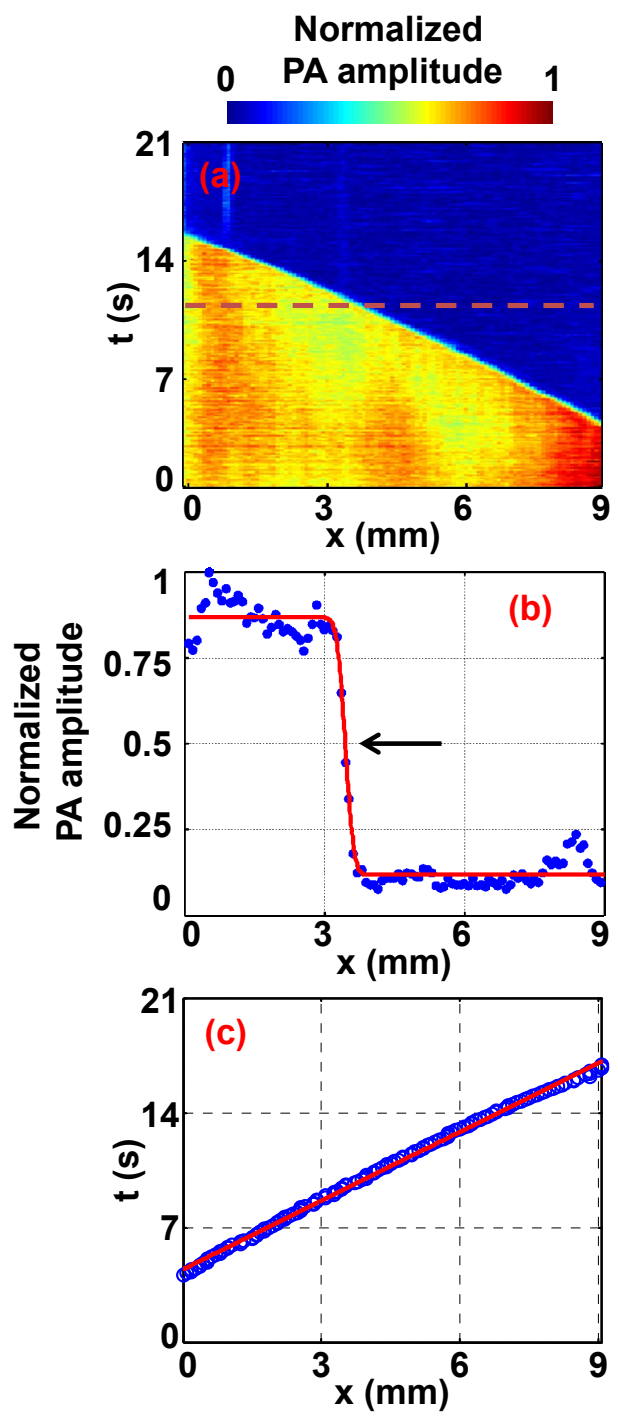

Fig. 2. Steps to extract the flow velocity. (a) Time course of the photoacoustic (PA) amplitude of the blood vessel. (b) Blue dots, one dimensional PA amplitude along the dashed line in (a); Red line, error function fitting; black arrow points to the mean value of the error function. The mean value represents the saline-blood-interface (SBI). (c) Blue circles, time course of the SBIs; red line, linear fitting. 
The flow velocity was extracted in three steps from the PA signals of the saline-blood interface (SBI). First, the whole process of the interface propagation through the field of view (FOV) of the transducer array was monitored. At each time point, a 2D image of the blood vessel was acquired and then converted to a one dimensional (1D) amplitude image via the Hilbert transformation, followed by taking the absolute value. Piecing together all the 1D images at consecutive time points, we obtained a final 2D amplitude image of the SBI, as shown in Fig. 2(a). Note that the $\mathrm{x}$-axis is the displacement along the blood vessel and the $\mathrm{y}$-axis is time. Second, at each time point, the 1D PA amplitude of the blood vessel was fitted by an error function to extract the location of the SBI, as indicated by the mean value of the error function in Fig. 2(b). Last, by tracking the SBI along the blood vessel, we calculated the blood stream flow velocity. As shown in Fig. 2(c), although only two measurements of the SBI sufficed to calculate the blood flow velocity, multiple measurements could improve the accuracy by linear fitting.
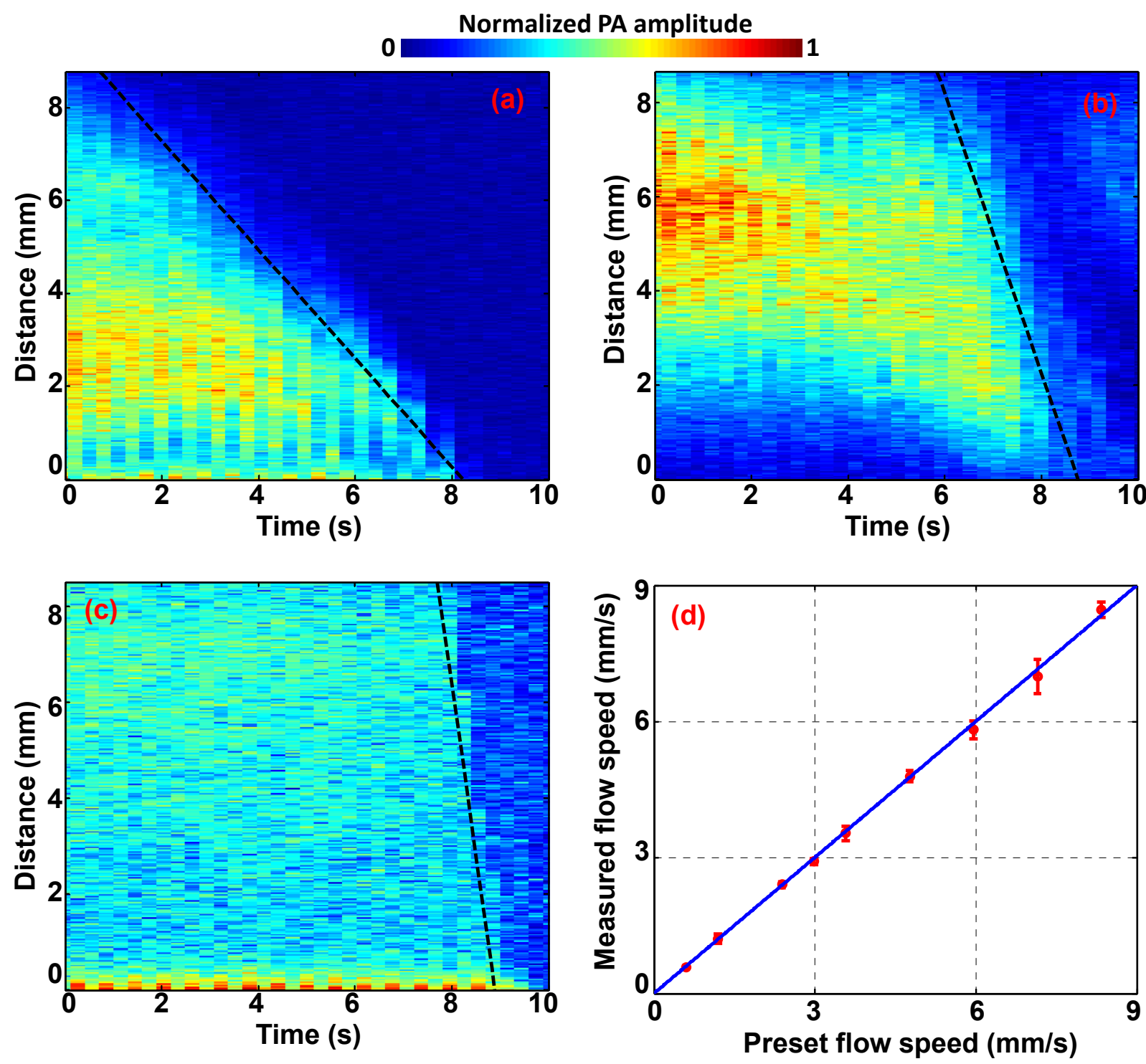

Fig. 3. Phantom experiments. Time course of the photoacoustic (PA) amplitude of the blood vessel with flow velocity at 0.6 (a), 1.2 (b), and $8 \mathrm{~mm} / \mathrm{s} \mathrm{(c).} \mathrm{Dashed} \mathrm{lines} \mathrm{indicate} \mathrm{the} \mathrm{linear} \mathrm{fitting} \mathrm{to} \mathrm{the} \mathrm{SBI.} \mathrm{(d)} \mathrm{Measured} \mathrm{flow} \mathrm{velocity} \mathrm{versus}$ preset flow velocity. Red circles, experimental measurement; blue line, ideal fit if the measured and preset velocities are identical. 


\section{PHANTOM EXPERIMENTS}

Phantom experiments were first performed to demonstrate the capability of our method in flow measurement. To confirm that our method can measure blood flow in biological tissue at depths in the optical diffusive regime, ${ }^{25}$ fresh chicken breast tissue with a thickness of $\sim 2 \mathrm{~mm}$ was laid atop the phantom blood vessel. Figs. 3(a-c) show three representative 2D amplitude images of the SBI, with blood stream flow velocities of $0.2,1.3$, and $4.5 \mathrm{~mm} / \mathrm{s}$. Because of the strong optical absorption difference between blood and saline, SBIs were imaged by PAT with high contrast, as shown in Figs. 3(a-c). Thus, our measured blood flow velocities, obtained from linear fittings of the SBI spatialtemporal locations, agreed well with the preset values, as shown in Fig. 3(d). The root-mean-squared error of prediction (RMSEP) $)^{26}$ was calculated to be $0.29 \mathrm{~mm} / \mathrm{s}$, indicating that our method can measure deep blood flow with high accuracy. Thus, we conclude that our method should be able to measure blood flow velocity in vivo.

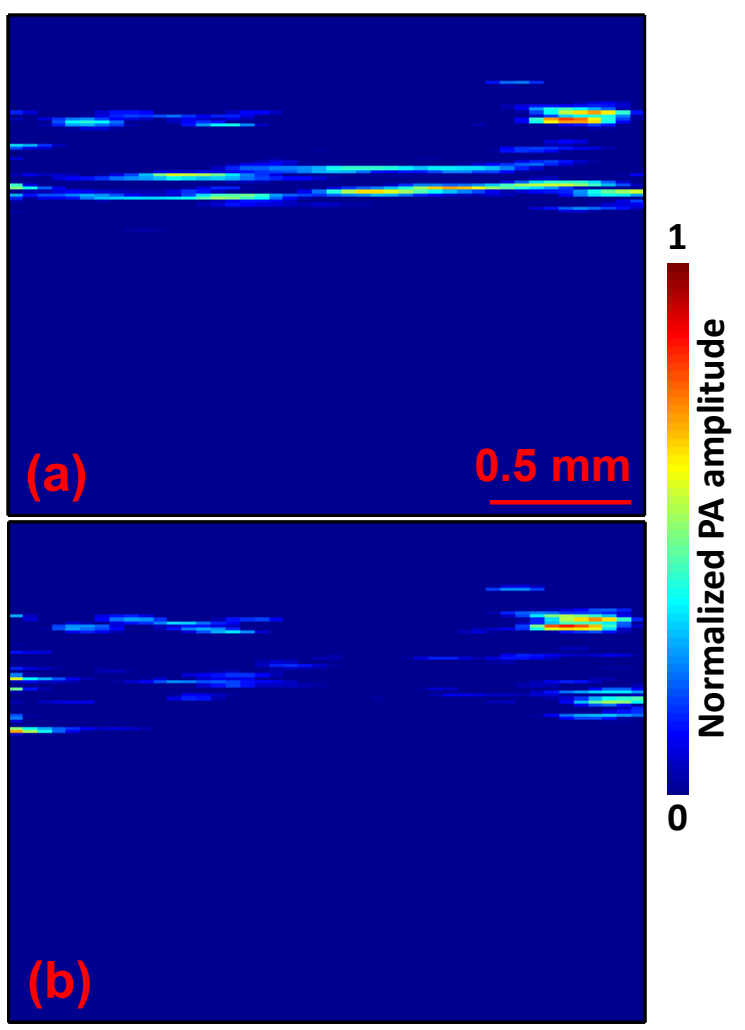

Fig. 4. In vivo experiments. Photoacoustic images of the tail vein before saline injection (a) and after saline injection (b).

\section{IN VIVO EXPERIMENTS}

To confirm the detection ability of the saline-injection-based method, we then measured blood flow velocity in a mouse tail vein in vivo. Again, a slice of chicken breast tissue $(\sim 1 \mathrm{~mm})$ was put atop the tail vein to increase the measurement depth. Saline was injected into the tail vein with a home-made catheter to induce the SBI for blood flow measurement. The distance between the injection spot and the imaging window was $\sim 3 \mathrm{~cm}$. During the experiment, an infrared lamp kept the mouse warm, and a breathing anesthesia system (E-Z Anesthesia, Euthanex) kept the mouse motionless. All experimental animal procedures were carried out in conformity with laboratory animal protocols approved by the Animal Studies Committee of Washington University in St. Louis. As shown in Fig. 4(a), before saline injection, the whole tail vein in the field of view (FOV) could be clearly observed. However, when the saline flushed in, there were almost no signals from the blood vessel anymore because of the low optical 
absorption of saline, as shown in Fig. 4(b). Based on the same procedure as in the phantom experiments, the blood flow velocity was quantified to be around $4.5 \mathrm{~mm} / \mathrm{s}$.

\section{DISCUSSION AND CONCLUSIONS}

In summary, to our knowledge, for the first time we achieved deep blood flow measurement in vivo with AR-PAT. SBIs were created by injecting saline into the blood vessel, and the blood flow velocity was measured by monitoring a single SBI propagation in the blood stream. Our phantom experiments showed that we could achieve a highly accurate measurement of blood flow velocity in deep biological tissue, with a RMSEP of $0.29 \mathrm{~mm} / \mathrm{s}$. The in vivo mouse experiments indicated that we could potentially measure blood flow in humans. Based on saline injection, our method is particularly suitable for blood flow quantification in patients already undergoing intravenous infusion. Considering the difficulty that ultrasound encounters in measuring slow blood flow and the oxygen saturation of hemoglobin $\left(\mathrm{sO}_{2}\right)$, PA-based deep flow and $\mathrm{sO}_{2}$ measurement opens a window for $\mathrm{MRO}_{2}$ quantification in humans, which may lead to significant applications such as noninvasive tumor screening and blood disorder diagnosis.

\section{ACKNOWLEDGEMENTS}

The authors would like to thank Prof. James Ballard for manuscript editing. This work was sponsored in part by National Institutes of Health grants DP1 EB016986 (NIH Director's Pioneer Award), R01 CA186567 (NIH Director's Transformative Research Award), R01 EB016963, S10 RR026922, and R01 CA159959. L.W. has a financial interest in Microphotoacoustics, Inc. and Endra, Inc., which, however, did not support this work.

\section{REFERENCES}

[1] L. V. Wang and S. Hu, "Photoacoustic Tomography: In Vivo Imaging from Organelles to Organs," Science 335(6075), 1458-1462 (2012).

[2] J. Xia and L. V. Wang, "Small-Animal Whole-Body Photoacoustic Tomography: A Review," Ieee T BioMed Eng 61(5), 1380-1389 (2014).

[3] L. V. Wang, "Multiscale photoacoustic microscopy and computed tomography," Nat Photonics 3(9), 503509 (2009).

[4] P. Beard, "Biomedical photoacoustic imaging," Interface Focus 1(4), 602-631 (2011).

[5] V. Ntziachristos, "Going deeper than microscopy: the optical imaging frontier in biology," Nat Methods 7(8), 603-614 (2010).

[6] M. Tang, Y. Zhou, R. Zhang and L. V. Wang, "Noninvasive photoacoustic microscopy of methemoglobin in vivo," J. Biomed. Opt. 20(3), 036007 (2015).

[7] Y. Zhou, G. Li, L. Zhu, C. Li, L. A. Cornelius and L. V. Wang, "Handheld photoacoustic probe to detect both melanoma depth and volume at high speed in vivo," J. Biophotonics 1(7), (2015).

[8] Y. Zhou, W. Xing, K. I. Maslov, L. A. Cornelius and L. V. Wang, "Handheld photoacoustic microscopy to detect melanoma depth in vivo," Opt Lett 39(16), 4731-4734 (2014).

[9] W. Song, Q. Wei, W. Z. Liu, T. Liu, J. Yi, N. Sheibani, A. A. Fawzi, R. A. Linsenmeier, S. L. Jiao and H. F. Zhang, "A combined method to quantify the retinal metabolic rate of oxygen using photoacoustic ophthalmoscopy and optical coherence tomography," Sci Rep-Uk 4((2014).

[10] J. M. Yang, C. Favazza, R. Chen, J. Yao, X. Cai, K. Maslov, Q. F. Zhou, K. K. Shung and L. V. Wang, "Simultaneous functional photoacoustic and ultrasonic endoscopy of internal organs in vivo," Nat Med 18(8), 1297$+(2012)$.

[11] B. Ning, M. J. Kennedy, A. J. Dixon, N. D. Sun, R. Cao, B. T. Soetikno, R. M. Chen, Q. F. Zhou, K. K. Shung, J. A. Hossack and S. Hu, "Simultaneous photoacoustic microscopy of microvascular anatomy, oxygen saturation, and blood flow," Opt. Lett. 40(6), 910-913 (2015).

[12] W. Song, W. Liu and H. F. Zhang, "Laser-scanning Doppler photoacoustic microscopy based on temporal correlation," Appl Phys Lett 102(20), (2013). 
[13] Y. Zhou, J. Liang, K. I. Maslov and L. V. Wang, "Calibration-free in vivo transverse blood flowmetry based on cross correlation of slow time profiles from photoacoustic microscopy," Opt. Lett. 38(19), 3882-3885 (2013).

[14] C. Yeh, S. Hu, K. Maslov and L. H. V. Wang, "Photoacoustic microscopy of blood pulse wave," J. Biomed. Opt. 17(7), (2012).

[15] Z. Guo, L. Li and L. V. Wang, "On the speckle-free nature of photoacoustic tomography," Med Phys 36(9), 4084-4088 (2009).

[16] Z. Guo, Z. Xu and L. V. Wang, "Dependence of photoacoustic speckles on boundary roughness," J. Biomed. Opt. 17(4), (2012).

[17] J. Yao, K. I. Maslov, Y. Shi, L. A. Taber and L. V. Wang, "In vivo photoacoustic imaging of transverse blood flow by using Doppler broadening of bandwidth," Opt. Lett. 35(9), 1419-1421 (2010).

[18] Y. Zhou, J. Yao, K. I. Maslov and L. V. Wang, "Calibration-free absolute quantification of particle concentration by statistical analyses of photoacoustic signals in vivo," J Biomed Opt 19(3), 37001 (2014).

[19] S. Chen, T. Ling, S. W. Huang, H. W. Baac and L. Guo, "Photoacoustic correlation spectroscopy and its application to low-speed flow measurement," Opt. Lett. 35(8), 1200-1202 (2010).

[20] H. Fang, K. Maslov and L. V. Wang, "Photoacoustic doppler effect from flowing small light-absorbing particles," Phys Rev Lett 99(18), (2007).

[21] L. Wang, J. Xia, J. Yao, K. I. Maslov and L. V. Wang, "Ultrasonically Encoded Photoacoustic Flowgraphy in Biological Tissue," Phys Rev Lett 111(20), (2013).

[22] A. Needles, A. Heinmiller, J. Sun, C. Theodoropoulos, D. Bates, D. Hirson, M. Yin and F. S. Foster, "Development and Initial Application of a Fully Integrated Photoacoustic Micro-Ultrasound System," Ieee T Ultrason Ferr 60(5), 888-897 (2013).

[23] Y. Zhou, J. Liang, and L. V. Wang, "Cuffing-based photoacoustic flowmetry in humans in the optical diffusive regime," J. Biophotonics 1-5 (2015), DOI 10.1002.

[24] Y. Zhou, J. Poudel, G. Li, and L. V. Wang, "In vivo photoacoustic flowmetry at depths of the diffusive regime based on saline injection," J. Biomed. Opt. 20(8), 087001 (2015).

[25] L. V. Wang and H. Wu, Biomedical Optics: Principles and Imaging, WILEY (2007).

[26] Y. Zhou, C. Zhang, D. K. Yao and L. V. Wang, "Photoacoustic microscopy of bilirubin in tissue phantoms," J. Biomed. Opt. 17(12), (2012). 